\title{
The Blood-Brain Barrier and Selective Vulnerability in Experimental Thiamine-Deficiency Encephalopathy in the Mouse
}

\section{Nobutoshi Harata and Yuzo Iwasaki}

On page 64 of the above article, Figure 2 was inadvertantly omitted. It appears below with legend.

A

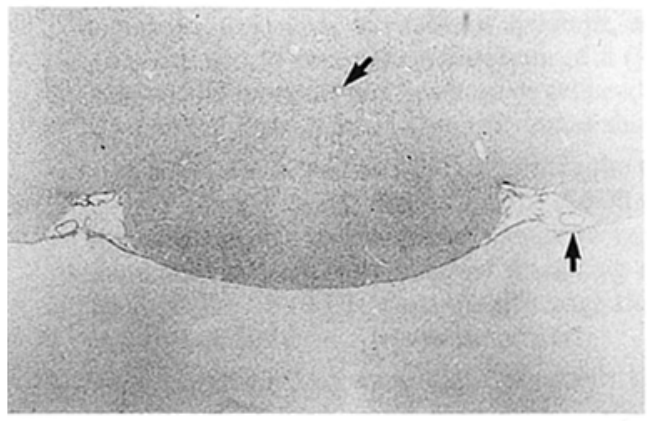

C

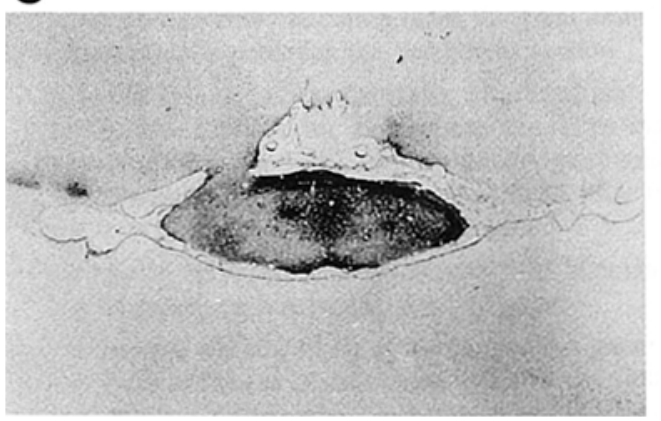

B

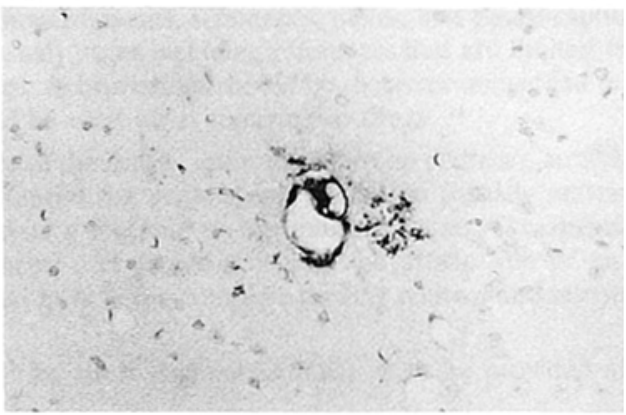

D

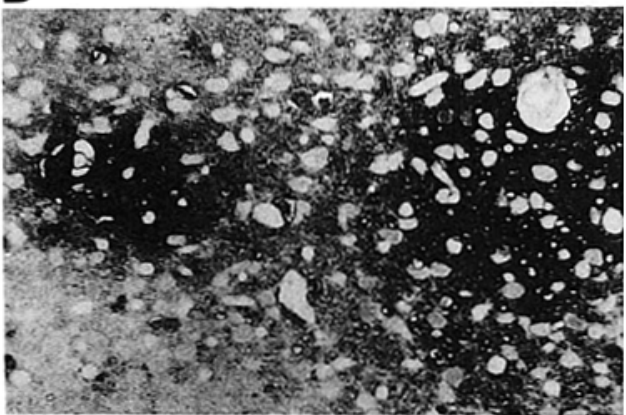

Figure 2. Immunohistochemistry for autologous albumin. A: control animal of Group C sacrificed on day 10 . The arrows point to negative staining of the vascular lumina. $B$ : high-power view of the mamillary nucleus of the mouse in Group PT on day 9. The section was stained for autologous albumin. Note the perivascular confinement of albumin extravasation. $C$ : low-power view of the mamillary nucleus of the mouse in Group PT on day 10 , when the neurological signs were manifest. The extent of albumin leakage is clearly demonstrated. $D$; high-power view of the same area as in $C$. The extravasated albumin is distributed more diffusely and widely in the affected area than on day $9(B)$.

\section{1}

0885-7490/96/0900-0281\$09.50/0 @ 1996 Plenum Publishing Corporation 